\title{
Biological and clinical implications of BIRC3 mutations in chronic lymphocytic leukemia
}

Haematologica 2020

Volume 105(2):448-456

\section{Correspondence:}

DAVIDE ROSSI

davide.rossi@eoc.ch

GIANLUCA GAIDANO

gianluca.gaidano@med.uniupo.it

Received: March 29, 2019.

Accepted: July 24, 2019.

Pre-published: August 1, 2019.

doi:10.3324/haematol.2019.219550

Check the online version for the most updated information on this article, online supplements, and information on authorship \& disclosures: www.haematologica.org/content/105/2/448

\section{(C)2020 Ferrata Storti Foundation}

Material published in Haematologica is covered by copyright. All rights are reserved to the Ferrata Storti Foundation. Use of published material is allowed under the following terms and conditions:

https://creativecommons.org/licenses/by-nc/4.0/legalcode. Copies of published material are allowed for personal or internal use. Sharing published material for non-commercial purposes is subject to the following conditions:

https://creativecommons.org/licenses/by-nc/4.0/leǵalcode, sect. 3. Reproducing and sharing published material for commercial purposes is not allowed without permission in writing from the publisher.
Fary Diop,${ }^{1 *}$ Riccardo Moia,${ }^{1 *}$ Chiara Favini, ${ }^{1}$ Elisa Spaccarotella,${ }^{1}$ Lorenzo De Paoli, ${ }^{1}$ Alessio Bruscaggin, ${ }^{2}$ Valeria Spina, ${ }^{2}$ Lodovico Terzi-di-Bergamo, ${ }^{2}$

Francesca Arruga, ${ }^{3}$ Chiara Tarantelli, ${ }^{4}$ Clara Deambrogi, ${ }^{1}$ Silvia Rasi, ${ }^{1}$ Ramesh Adhinaveni, ${ }^{1}$ Andrea Patriarca, ${ }^{1}$ Simone Favini,${ }^{1}$ Sruthi Sagiraju, ${ }^{1}$ Clive Jabangwe, ${ }^{1}$ Ahad A. Kodipad, ${ }^{1}$ Denise Peroni, ${ }^{1}$ Francesca R. Mauro, ${ }^{5}$ Ilaria Del Giudice, ${ }^{5}$ Francesco Forconi, ${ }^{6,7}$ Agostino Cortelezzi, ${ }^{8}$ Francesco Zaja, ${ }^{9}$ Riccardo Bomben, ${ }^{10}$ Francesca Maria Rossi, ${ }^{10}$ Carlo Visco, ${ }^{11}$ Annalisa Chiarenza, ${ }^{12}$ Gian Matteo Rigolin, ${ }^{13}$ Roberto Marasca, ${ }^{14}$ Marta Coscia, ${ }^{15}$ Omar Perbellini, ${ }^{16}$ Alessandra Tedeschi, ${ }^{17}$ Luca Laurenti, ${ }^{18}$ Marina Motta, ${ }^{19}$ David Donaldson, ${ }^{20}$ Phil Weir, ${ }^{20}$ Ken Mills, ${ }^{21}$ Patrick Thornton, ${ }^{22}$ Sarah Lawless, ${ }^{20}$ Francesco Bertoni, ${ }^{4}$ Giovanni Del Poeta, ${ }^{23}$ Antonio Cuneo, ${ }^{13}$ Antonia Follenzi, ${ }^{24}$ Valter Gattei, ${ }^{10}$ Renzo Luciano Boldorini, ${ }^{25}$ Mark Catherwood, ${ }^{20}$ Silvia Deaglio, ${ }^{3}$ Robin Foà, ${ }^{5}$ Gianluca Gaidano ${ }^{\circ}$ and Davide Rossi ${ }^{\circ}$

${ }^{1}$ Division of Hematology, Department of Translational Medicine, Amedeo Avogadro University of Eastern Piedmont, Novara, Italy; ${ }^{2}$ nstitute of Oncology Research and Oncology Institute of Southern Switzerland, Bellinzona, Switzerland; ${ }^{3}$ Department of Medical Sciences, University of Turin \& Italian Institute for Genomic Medicine, Turin, Italy; ${ }^{4}$ Università della Svizzera Italiana, Institute of Oncology Research, Bellinzona, Switzerland; ${ }^{5} \mathrm{Hematology}$, Department of Cellular Biotechnologies and Hematology, Sapienza University, Rome, Italy; ${ }^{6}$ Cancer Sciences Unit, Southampton Cancer Research UK and National Institute for Health Research Experimental Cancer Medicine Centre, University of Southampton, Southampton, UK; 'Division of Hematology, University of Siena, Siena, Italy; ${ }^{8}$ Department of Hematology Oncology, Foundation IRCCS Ca' Granda Ospedale Maggiore Policlinico and University of Milan, Milan, Italy; ${ }^{9}$ Clinica Ematologica, DAME, University of Udine, Udine, Italy; ${ }^{10} \mathrm{Clinical}$ and Experimental Onco-Hematology Unit, Centro di

Riferimento Oncologico, Istituto di Ricovero e Cura a Carattere Scientifico (IRCCS), Aviano, Italy; ${ }^{11}$ Department of Cell Therapy and Hematology, Ospedale San Bortolo, Vicenza, Italy; ${ }^{12}$ Division of Hematology, Azienda Ospedaliera Universitaria Policlinico-OVE, Catania, Italy; ${ }^{13} \mathrm{Hematology}$ Section, Azienda Ospedaliero Universitaria Arcispedale S. Anna, University of Ferrara, Ferrara, Italy; ${ }^{14}$ Division of Hematology, Department of Oncology and Hematology, University of Modena and Reggio Emilia, Modena, Italy; ${ }^{15}$ Division of Hematology, Azienda Ospedaliero Universitaria Città della Salute e della Scienza and University of Turin, Turin, Italy; ${ }^{16}$ Section of Hematology, Department of Medicine, University of Verona, Verona, Italy; ${ }^{17}$ Department of Oncology/Haematology, Niguarda Cancer Center, Niguarda Ca Granda Hospital, Milan, Italy; ${ }^{18}$ Fondazione Policlinico Universitario A. Gemelli, Rome, Italy; ${ }^{19}$ Department of Hematology, Spedali Civili, Brescia, Italy; ${ }^{20} \mathrm{Clinical}$ Haematology, Belfast City Hospital, Belfast Health and Social Care Trust, Belfast, Northern Ireland, UK; ${ }^{21}$ Centre for Cancer Research and Cell Biology (CCRCB), Queen's University Belfast, Belfast, Northern Ireland, UK; ${ }^{22}$ Department of Haematology, Beaumont Hospital, Dublin, Ireland; ${ }^{23}$ Department of Hematology, Tor Vergata University, Rome, Italy; ${ }^{24}$ Department of Health Sciences, University of Eastern Piedmont Amedeo Avogadro, Novara, Italy; ${ }^{25}$ Department of Pathology, University of Eastern Piedmont Amedeo Avogadro, Novara, Italy.

$\star * F D$ and $R M$ contributed equally to this work

${ }^{\circ} G G$ and $D R$ contributed equally to this work.

\section{ABSTRACT}

IRC3 is a recurrently mutated gene in chronic lymphocytic leukemia R (CLL) but the functional implications of BIRC3 mutations are largely 1 unexplored. Furthermore, little is known about the prognostic impact of BIRC3 mutations in CLL cohorts homogeneously treated with first-line fludarabine, cyclophosphamide, and rituximab (FCR). By immunoblotting analysis, we showed that the non-canonical nuclear factor- $\kappa \mathrm{B}$ pathway is active in BIRC3-mutated cell lines and in primary CLL samples, as documented by the stabilization of MAP3K14 and by the nuclear localization of p52. In addition, BIRC3-mutated primary CLL cells are less sensitive to fludarabine. In order to confirm in patients that BIRC3 mutations confer resistance to fludarabine-based chemoimmunotherapy, a retrospective multicenter cohort of 287 untreated patients receiving first-line FCR was analyzed 
by targeted next-generation sequencing of 24 recurrently mutated genes in CLL. By univariate analysis adjusted for multiple comparisons BIRC3 mutations identify a poor prognostic subgroup of patients in whom FCR treatment fails (median progression-free survival: 2.2 years, $P<0.001$ ) similar to cases harboring TP53 mutations (median progression-free survival: 2.6 years, $P<0.0001$ ). BIRC3 mutations maintained an independent association with an increased risk of progression with a hazard ratio of $2.8(95 \%$ confidence interval 1.4-5.6, $P=0.004$ ) in multivariate analysis adjusted for TP53 mutation, $17 \mathrm{p}$ deletion and IGHV mutation status. If validated, BIRC3 mutations may be used as a new molecular predictor to select high-risk patients for novel frontline therapeutic approaches.

\section{Introduction}

Nuclear factor- $\mathrm{\kappa B}$ (NF- $\mathrm{KB}$ ) signaling is a key component of the development and evolution of chronic lymphocytic leukemia (CLL). ${ }^{1}$ Two NF-kB pathways exist, namely the canonical and non-canonical pathways. ${ }^{2}$ The former is triggered by B-cell receptor signaling via Bruton tyrosine kinase (BTK), while the latter is activated by members of the tumor necrosis factor (TNF) cytokine family. ${ }^{3}$ Upon receptor binding, the TRAF3/MAP3K14-TRAF2/BIRC3 negative regulatory complex of non-canonical NF-kB signaling is disrupted, MAP3K14 (also known as NIK), the central activating kinase of the pathway, is released and activated to induce the phosphorylation and proteasomal processing of $\mathrm{p} 100$, thereby leading to the formation of p52-containing NF- $\mathrm{KB}$ dimers. The p52 protein dimerizes with RelB to translocate into the nucleus, where it regulates gene transcription. BIRC3 (Baculoviral IAP Repeat Containing 3 ) is a negative regulator of non-canonical NF$\kappa B$. Physiologically, BIRC3 (also known as cIAP2) catalyzes MAP3K14 protein ubiquitination in a manner that is dependent on the $\mathrm{E} 3$ ubiquitinine ligase activity of its Cterminal RING domain. MAP3K14 ubiquitination results in its proteasomal degradation. ${ }^{4}$

B-cell neoplasia often pirates signaling pathways by molecular lesions to promote survival and proliferation. Although according to bioinformatics criteria BIRC3 is one of the candidate driver genes of CLL, the functional implications of BIRC3 mutations are partially unexplored. ${ }^{5-7}$ Furthermore, little is known about the prognostic impact of BIRC3 mutations in CLL cohorts homogeneously treated first-line with fludarabine, cyclophosphamide, and rituximab (FCR).

FCR is the most effective chemoimmunotherapy regimen for the management of CLL in young and fit patients devoid of TP53 disruption. ${ }^{8}$ Survival after FCR is, however, variable, and is affected by the molecular characteristics of the CLL clone. ${ }^{9}$ Deletion of 17p and TP53 mutations are present in most, but not all patients who are refractory to chemo-immunotherapy, which prompts the identification of additional biomarkers associated with early failure of FCR. ${ }^{10-12}$

\section{Methods}

\section{Functional studies}

The human CLL cell line MEC1, the splenic marginal zone lymphoma cell lines SSK41 and VL51, the mantle cell lymphoma cell lines MAVER-1, Z-138 and JEKO-1, the human HEK-293T cell line, as well as primary CLL cells were used in functional experiments. The entire non-canonical NF-kB pathway was assessed by western blot analysis. Quantitative real-time polymerase chain reaction
(qRT-PCR) was utilized to analyze the non-canonical NF-KB signature. Primary CLL were exposed to fludarabine and venetoclax for 24-48 $\mathrm{h}$ and apoptosis was measured using the eBioscience Annexin V Apoptosis Detection Kit APC (ThermoFisher). Details are supplied in the Online Supplementary Methods.

\section{Cancer personalized profiling by deep sequencing}

A retrospective multicenter cohort of 287 untreated CLL patients receiving first-line therapy with FCR was analyzed for mutations, including 173 patients from a previously published multicenter clinical series and 114 new patients not included in our previous report. ${ }^{10}$ The study was approved by the Ethical Committee of the Ospedale Maggiore della Carità di Novara associated with the Amedeo Avogadro University of Eastern Piedmont (study number CE 67/14). Further information is provided in the Online Supplementary Methods. A targeted resequencing gene panel was designed to include: (i) coding exons plus splice site of 24 genes known to be implicated in CLL pathogenesis and/or prognosis; (ii) 3'UTR of NOTCH1; and (iii) enhancer and promoter region of PAX5 (size of the target region: 66627bp) (Table S1). ${ }^{67}$ The next-generation sequencing libraries for genomic DNA (gDNA) were constructed using the KAPA Library Preparation Kit (Kapa Biosystems) and those for RNA were constructed using the RNA Hyper Kit (Roche). Multiplexed libraries ( $\mathrm{n}=10$ per run) were sequenced using 300-bp paired-end runs on a MiSeq sequencer (Illumina) to obtain a coverage of at least $2000 \mathrm{x}$ in $>90 \%$ of the target region (66627 bp) in 80\% of cases (Online Supplementary Table S2). A robust and previously validated bioinformatics pipeline was used for variant calling (Online Supplementary Appendix).

\section{Statistical analysis}

Progression-free survival (PFS) was the primary endpoint. Survival analysis was performed with the Kaplan-Meier method and compared between strata using the log-rank test. To account for multiple testing, adjusted $P$ values were calculated using the Bonferroni correction. The adjusted association between exposure variables and PFS was estimated by Cox regression. Internal validation of the multivariate analysis was performed using a bootstrap approach. Statistical significance was defined as a $P$ value $<0.05$ (Online Supplementary Appendix).

\section{Results}

BIRC3 mutations associate with activation of non-canonical nuclear factor- $\kappa \mathrm{B}$ signaling

In order to map unique BIRC3 mutations in CLL comprehensively, we compiled somatically confirmed variants identified in the current CLL study cohort with those identified in previous studies ${ }^{13}$ or listed in public CLL mutation catalogues (Figure 1A). Virtually all BIRC3 mutations were frameshift mutations or stop codons clustering in two hotspot regions comprised between amino acids 
367-438 and amino acids 537-564. BIRC3 variants were predicted to generate aberrant truncated transcripts causing the elimination or truncation of the C-terminal RING domain of the BIRC3 protein. The RING domain of
BIRC3 harbors the E3 ubiquitin ligase activity that is essential for proteasomal degradation of MAP3K14, the central activating kinase of non-canonical NF- $\mathrm{KB}$ signaling. This observation points to non-canonical NF-кB activation
A

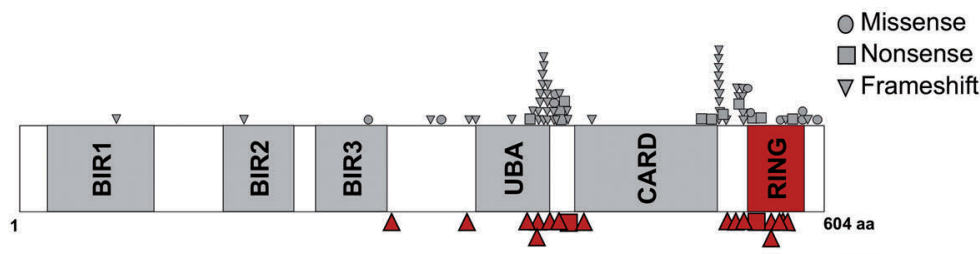

$\square$ Nonsense

B

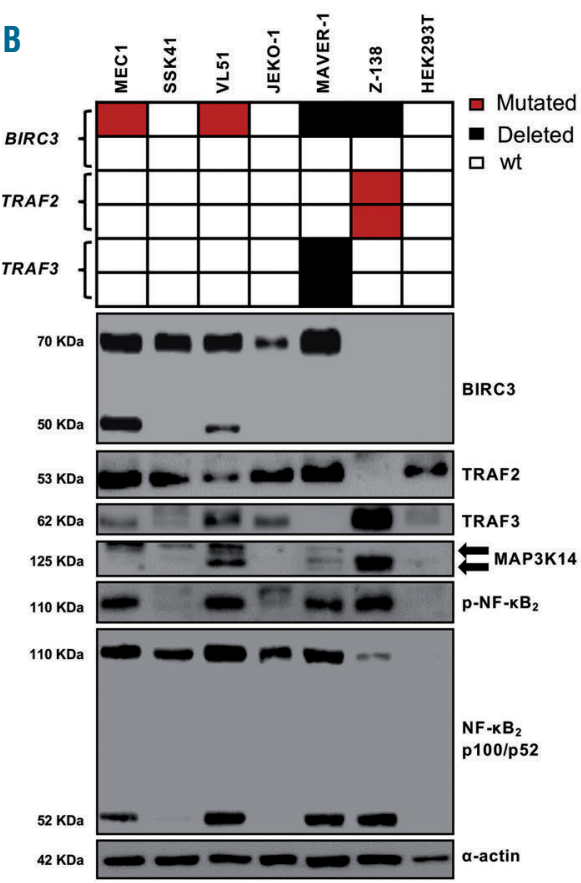

C

$\Delta$ Frameshift

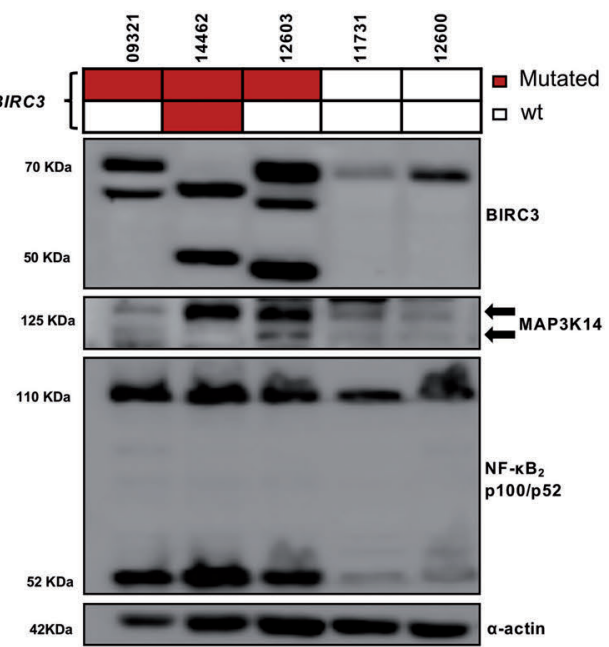

D

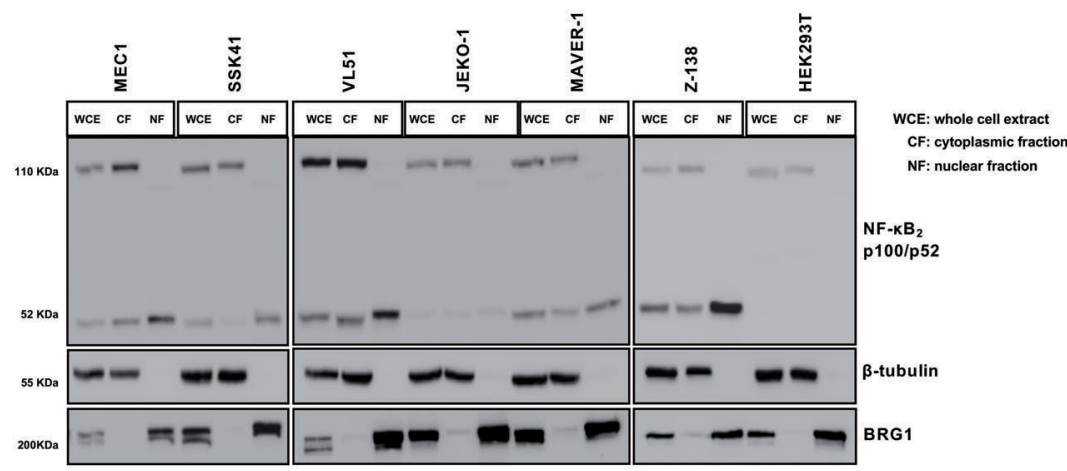

E

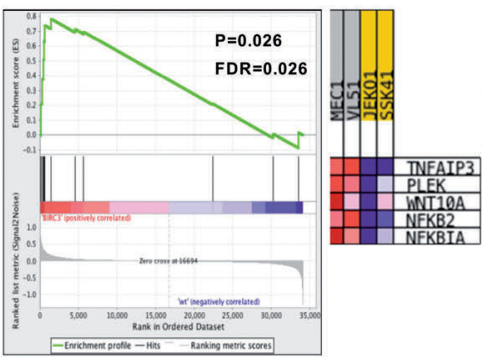

$\mathbf{F}$

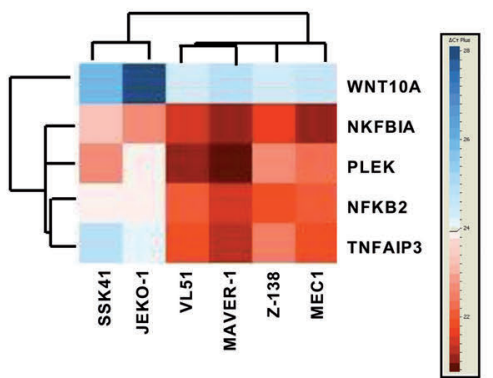

Figure 1. The non-canonical nuclear factor-KB pathway is active in BIRC3mutated chronic lymphocytic leukemia cell lines and primary samples. (A) Disposition of BIRC3 mutations across the protein. The mutations identified by Landau et al. ${ }^{6}$, Puente et al. ${ }^{7}$ and from a public CLL mutation catalogue (COSMIC v85) are plotted in gray. Individual BIRC3 mutations identified in the current studied cohort and in our previous study ${ }^{13}$ are plotted in red. (B) Western blot analysis of BIRC3 protein expression and NF- $\mathrm{KB}_{2}$ activation and processing in the splenic marginal zone lymphoma (SMZL) cell lines SSK41, VL51 and in the chronic lymphocytic leukemia (CLL) cell line MEC1, carrying wildtype (wt) or disrupted BIRC3. The MAVER-1 and Z-138 cell lines were used as positive controls of non-canonical NF-KB activation, harboring genetic activation of non-canonical NF-KB signaling. The JEKO-1 and HEK 293T cell lines were used as negative controls for noncanonical NF- $\mathrm{KB}$ signaling. $\alpha$-actin was used as a loading control. Color codes indicate the gene status in each cell line. The aberrant BIRC3 band expressed in MEC1 and VL51 cell lines corresponds in size to the predicted BIRC3-truncated protein, encoded by the mutant allele. (C) Western blot analysis showing BIRC3 expression and $\mathrm{NF}-\mathrm{KB}_{2}$ processing in purified primary tumor cells from five CLL and SMZL patients carrying wildtype or disrupted BIRC3. Color codes indicate the gene status in each cell line. The aberrant BIRC3 bands in patients 09321, 14462 and 12603 correspond in size to the predicted BIRC3-truncated protein encoded by the mutant allele. $\alpha$-actin was used as a loading control. (D) Western blot of whole cell extract, cytoplasmic or nuclear fractions of the SMZL and CLL cell lines probed for the NF-KB2 subunits p100 and p52. The MAVER-1 and Z-138 cell lines served as positive controls while the JEKO-1 and HEK 293T cell lines were used as negative controls. $\beta$-tubulin and BRG1 served as controls for the purity of the cytoplasmic and nuclear fractionations, respectively. (E) Gene set enrichment analysis score and distribution of noncanonical NF- $\mathrm{KB}$ target genes along the rank of transcripts differentially expressed in the SMZL cell lines SSK41, VL51 and in the CLL cell line MEC1. The JEKO-1 cell line was used as a negative control. (F) Validation of expression of non-canonical NF-KB target genes in the same SMZL and CLL cell lines as determined by quantitative real-time poly merase chain reaction analysis. Changes of gene expression were nor malized to GAPDH expression; relative quantities were $\log _{2}$ normalized to control samples (the mantle cell lymphoma cell line, JEKO-1). 
through MAP3K14 stabilization as the predicted functional consequence of BIRC3 mutations in CLL.

The non-canonical NF- $\mathrm{kB}$ signaling was profiled by immunoblotting in B-cell tumor cell lines and primary CLL cells with different genetic make-up in the non-canonical NF- $\mathrm{KB}$ pathway to verify whether BIRC3 mutations lead to constitutive non-canonical NF-kB activation. Additional genetic features of the above-mentioned cell lines and primary CLL cells are shown in Online Supplementary Table S3. In the VL51 splenic marginal zone lymphoma cell line and in the MEC1 CLL cell lines, both harboring endogenous truncating mutations of the BIRC3 gene, non-canonical NF-KB signaling was constitutively active, as documented by the stabilization of MAP3K14, phosphorylation of $\mathrm{NF}-\mathrm{KB}_{2}$, its processing from $\mathrm{p} 100$ to $\mathrm{p} 52$, as well as the nuclear localization of p52 (Figure 1B-D). Consistent with the biochemical clues of non-canonical NF-KB activation, the gene expression signature of the VL51 and MEC1 cell lines was significantly enriched in non-canonical NF$\kappa \mathrm{B}$ target genes (Figure 1E, F). Non-canonical NF-кB signaling in BIRC3-mutated cells was consistent with that in mantle cell lymphoma cell lines known to harbor a disrupted TRAF3/MAP3K14-TRAF2/BIRC3 negative regulatory complex by loss of TRAF3 or TRAF2.${ }^{14}$ Like BIRC3mutated cell lines, primary CLL samples harboring inactivating mutations of $B I R C 3$ also showed stabilization of MAP3K14 and NF- $\mathrm{KB}_{2}$ processing from p100 to p52 (Figure $1 \mathrm{C})$, thus confirming that non-canonical NF-KB activation is also a feature of primary cells harboring BIRC 3 variants. MAP3K14 stabilization is largely associated with BIRC3 mutations. Indeed all seven cases harboring non-canonical NF- $\mathrm{KB}$ genetic lesions showed either a strong or a slight MAP3K14 band, while, conversely, only one out of five cases lacking a non-canonical NF-KB lesion had MAP3K14 expression (Fisher exact test, $P=0.01$ ).

MAP3K14 was genetically targeted by shRNA to test whether BIRC3-mutated cells are addicted to its stabilization. Compared to non-targeting shRNA, the most efficient anti-MAP3K14 shRNA-D resulted in a partial silencing of MAP3K14 and in decreased NF- $\mathrm{KB} 2$ processing from p100 to p52. This translated into a reduced cell viability of the BIRC3-mutated VL51 cell line transduced with shRNA-D. This observation indicates that MAP3K14 stabilization is a vulnerability of BIRC3-mutated cells (Figure 2). In order to test the contribution of BTK to non-canonical NF-kB signaling when it is activated through BIRC3 mutations, BIRC3-mutated cell lines, as well as cell lines harboring a disrupted or competent TRAF3/MAP3K14TRAF2/BIRC3 negative regulatory complex were treated with ibrutinib at different dosages and non-canonical NF$\kappa \mathrm{B}$ signaling activation was probed by immunoblotting of the NF- $-\mathrm{B}_{2}$ processing from $\mathrm{p} 100$ to $\mathrm{p} 52$. Processing from p100 to p52 was unaffected by ibrutinib treatment in cell lines harboring BIRC3 mutations (Figure 3 ) or a disrupted TRAF3/MAP3K14-TRAF2/BIRC3 negative regulatory complex, consistent with the notion that BIRC3 mutations activate non-canonical NF- $\mathrm{kB}$ by bypassing BTK blockade by ibrutinib. ${ }^{14}$

\section{BIRC3 mutations confer resistance to fludarabine in primary chronic lymphocytic leukemia cells}

We performed in vitro pharmacological studies on primary CLL cells to verify the vulnerabilities of BIRC3mutated cells. CLL cells purified from patients carrying BIRC3 mutations were treated with increasing doses of fludarabine. Drug-induced apoptosis was compared to that of samples harboring TP53 mutations, which represent a control for fludarabine resistance. CLL cells devoid of genetic lesions in either BIRC3 or TP53 were used as a control for fludarabine sensitivity. The molecular characteristic of the ex-vivo CLL cells are listed in Online Supplementary Table S4.

BIRC3-mutated cells showed delayed fludarabineinduced cell death, as no response was observed after $24 \mathrm{~h}$ of treatment, at variance with TP53- and BIRC3-wildtype samples. At this time point, cell viability curves of BIRC3mutated samples overlapped almost completely with those of TP53-disrupted samples, which are known to be fludarabine resistant (Figure 4A). At $48 \mathrm{~h}$, the viability of BIRC3-mutated cells was lower than that of TP53-mutated samples, but higher than that of TP53-and BIRC3-wildtype samples (Figure 4B).
A

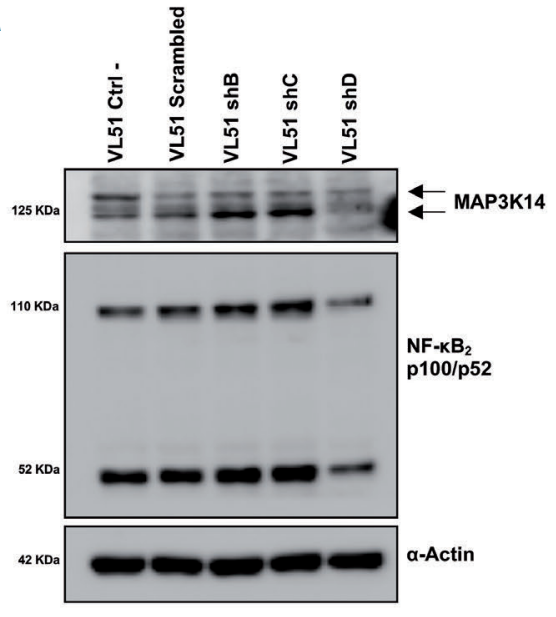

B

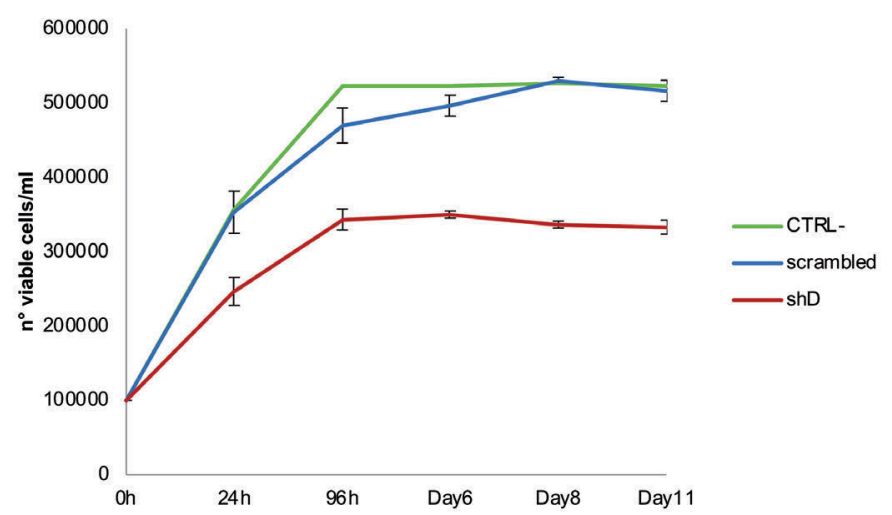

Figure 2. Knockdown of MAP3K14 by RNA interference in VL51 cells. (A) Western blot analysis for MAP3K14 expression and for NF-KB 2 processing of p100 to p52. (B) VL51 cell viability assessed by trypan blue after transduction with lentiviral vectors expressing the shRNA-D_MAP3K14 (shD: in red), a scrambled shRNA (scrambled: in blue), and in non-transfected cells (CTRL: in green). 
In order to assess whether BIRC3 mutations interfere with apoptosis, primary CLL cells were treated with venetoclax. Venetoclax treatment resulted in a similar reduction of cell viability in BIRC3-mutated cells, TP53-mutated cells and BIRC3/TP53-wildtype cells (Figure 4C, D). Such divergent sensitivity to fludarabine and venetoclax of BIRC3-mutated CLL cells indirectly suggests that BIRC3 mutations likely affect the upstream DNA damage response pathway rather than downstream apoptosis as a mechanism of inducing cell death.

\section{Patients harboring BIRC3 mutations are at risk of FCR failure}

In order to confirm in vivo in patients that BIRC3 mutations confer resistance to fludarabine-based chemoimmunotherapy, we correlated the BIRC3 mutation status with PFS of CLL patients treated with FCR. Mutational profiling was performed in 287 patients who received first-line FCR. The baseline features of the study cohort were consistent with progressive, previously untreated CLL (Table 1). The median follow-up was 6.8 years, with a median PFS and overall survival of 4.6 and 11.7 years, respectively (Table 1) consistent with observations in clinical trial cohorts. ${ }^{15}$ As expected, SF3B1 and NOTCH1 were the most frequently mutated genes identified in $13.9 \%$ and in $13.6 \%$ of patients, respectively, followed by TP53 in $9.4 \%$ and ATM in $6.9 \%$ of patients. BIRC3 was mutated in $3.1 \%$ of patients, reflecting the data reported in previous studies. ${ }^{6,7,13}$ Overall, 154/287 $(53.6 \%)$ cases harbored at least one non-synonymous somatic mutation in one of the 24 CLL genes included in our panel (range, 1-5 mutation per patient), which is consistent with the typical mutational spectrum of CLL requiring first-line treatment (Figure 5, Online Supplementary Table S5). ${ }^{6,7,16}$

By univariate analysis adjusted for multiple comparisons, among the genes analyzed in our panel, only TP53 mutations (median PFS of 2.6 years; $P<0.0001$ ) and BIRC3 mutations (median PFS of 2.2 years; $P<0.001$ ) (Figure 6A) were associated with significantly shorter PFS (Table 2). Following FCR treatment, the PFS of BIRC3-mutated patients was similar to that of cases har-
A
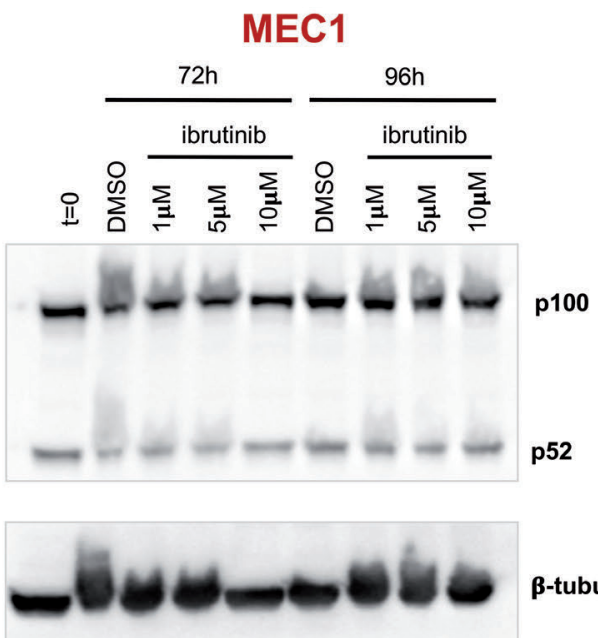

C

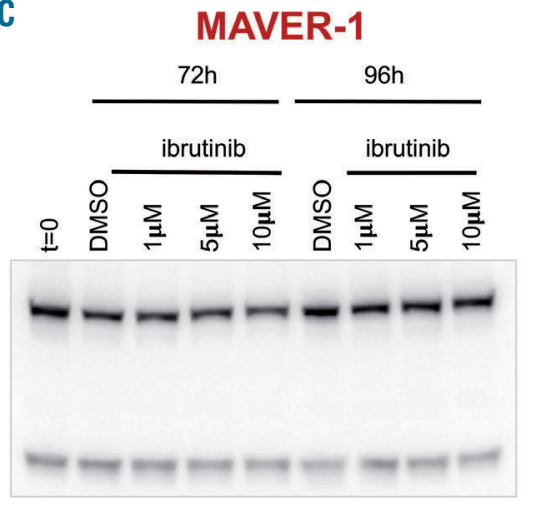

及-tubulin

D

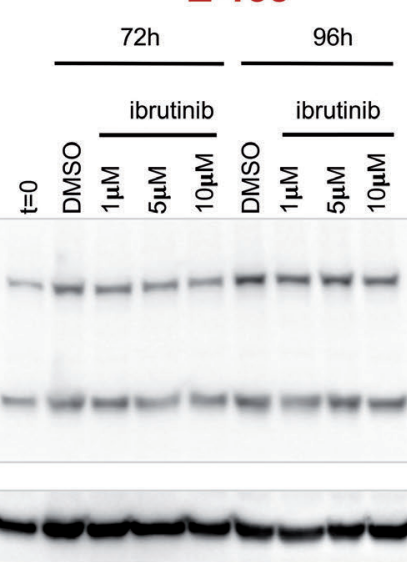

VL51

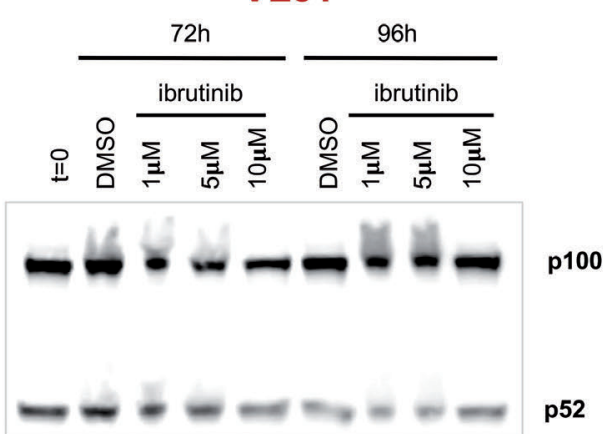

世
B

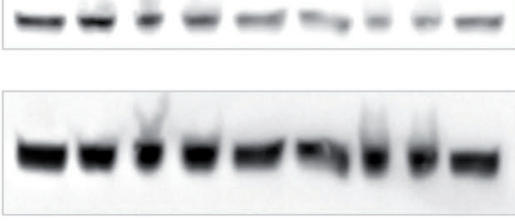

p52

E
JEKO-1

Figure 3. The non-canonical nuclear factor-кB pathway is not switched off by ibrutinib in BIRC3-mutated cell lines. Western blot showing $\mathrm{p} 100 / \mathrm{p} 52$ expression in (A) MEC1 and (B) VL51 cell lines that harbor BIRC3 mutations. (C) MAVER-1 and (D) Z-138 cell lines, known to be affected by non-canonical NF-KB pathway gene mutations and resistant to ibrutinib were used as positive controls. (E) The JEKO-1 cell line, known to be devoid of NF-KB pathway gene mutations and sensitive to ibrutinib, was used as a negative control. All cell lines were treated with different concentrations of ibrutinib for 72 and 96 h. DMSO: dimethylsulfoxide. 
boring TP53 disruption (Figure 6B). Consistently, BIRC3mutated patients had a lower likelihood of achieving complete response $(22.2 \%)$ at the end of FCR compared to BIRC3-wildtype cases $(76.7 \% ; P=0.001)$. Well-known molecular prognostic biomarkers of CLL, such as unmutated IGHV gene status and $17 \mathrm{p}$ deletion also associated with a significantly shorter PFS, supporting the representativeness of the study cohort (Table 2). By multivariate analysis including variables showing a multiplicityadjusted significant association with PFS, BIRC3 mutations maintained an independent association with PFS, with a hazard ratio of 2.8 ( $95 \%$ confidence interval: 1.45.6; $P=0.004$ ) (Table 2).

\section{Discussion}

The results of this study provide evidence that: (i) BIRC3 mutations are associated with activation of the noncanonical NF-KB pathway and with resistance to fludarabine in vitro; and (ii) BIRC3-mutated patients, like cases harboring TP53 disruption, are subject to failure of FCR chemoimmunotherapy.

The mere presence of somatic mutations is insufficient to implicate a gene in cancer. Cancer geneticists and bioinformaticians differentiate "passenger" events, likely being randomly acquired, to distinguish them from mutations targeting candidate "cancer-driver" genes, likely implicated in the tumor biology, according to a statistical definition. Any given gene is labeled as a candidate "cancer driver" if it harbors somatic point mutations at a statistically significant rate or pattern in cancer samples. In CLL, more than 40 genes fulfill the statistical definition of a candidate "cancer driver", including BIRC3, but few of them are biologically validated (i.e. SF3B1, NOTCH1, TP53, ATM, FBXW7). ${ }^{6,717-20}$ The BIRC3 gene codes for a protein that ubiquitinates and negatively regulates the central activating kinase of the non-canonical NF-kB pathway, namely MAP3K14 (also known as NIK). ${ }^{21,22}$ In lymphoid malignancies, the NF- $\mathrm{KB}$ pathway is a pivotal and positive mediator of cell proliferation and survival. ${ }^{5,23,24}$ With regards to CLL, BIRC3 mutations are absent in patients with monoclonal B-cell lymphocytosis, are rare at the time of diagnosis (3$4 \%)$, but are detectable in approximately $25 \%$ of fludarabine-refractory patients. ${ }^{13}$ In this study, we verified the biological consequences of BIRC3 mutations, showing that they are associated with activation of the non-canonical NF-KB pathway, that BIRC3-mutated lymphoid cells
A

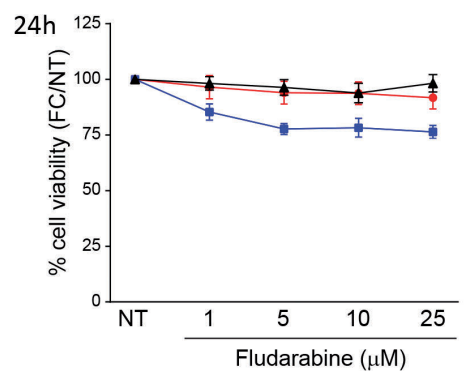

\begin{tabular}{|c|c|c|c|c|c|}
\cline { 2 - 6 } \multicolumn{1}{c|}{} & NT & $\begin{array}{c}\text { Fludarabine } \\
\mathbf{1 m M}\end{array}$ & $\begin{array}{c}\text { Fludarabine } \\
\mathbf{5 m M}\end{array}$ & $\begin{array}{c}\text { Fludarabine } \\
\mathbf{1 0 m M}\end{array}$ & $\begin{array}{c}\text { Fludarabine } \\
\mathbf{2 5 m M}\end{array}$ \\
\hline $\begin{array}{c}\text { TP53-M vs. } \\
\text { BIRC3-M }\end{array}$ & $P>0.9999$ & $P=0.9447$ & $P=0.8923$ & $P=0.9997$ & $P=0.4311$ \\
\hline $\begin{array}{c}\text { TP53-M vs. } \\
\text { WT }\end{array}$ & $P>0.9999$ & $P=0.0567$ & $P=0.0031$ & $P=0.0078$ & $P=0.0005$ \\
\hline $\begin{array}{c}\text { BIRC3-M } \\
\text { vs. WT }\end{array}$ & $P>0.9999$ & $P=0.1117$ & $P=0.0112$ & $P=0.0115$ & $P=0.0184$ \\
\hline
\end{tabular}

C

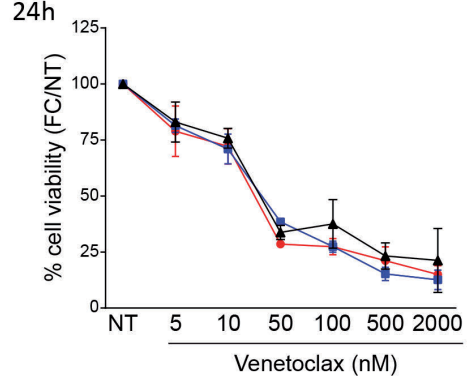

— TP53-M; BIRC3-WT

$\rightarrow$ TP53-WT; BIRC3-M

- TP53-WT; BIRC3-W7

B $48 \mathrm{~h}$

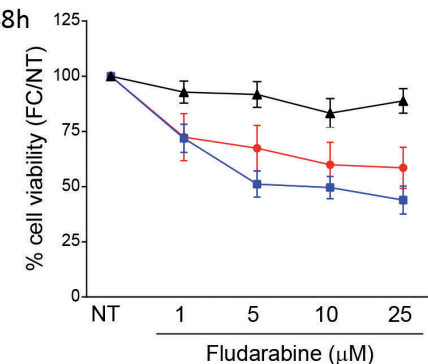

\begin{tabular}{|c|c|c|c|c|}
\hline NT & $\begin{array}{c}\text { Fludarabine } \\
\mathbf{1 m M}\end{array}$ & $\begin{array}{c}\text { Fludarabine } \\
\mathbf{5 m M}\end{array}$ & $\begin{array}{c}\text { Fludarabine } \\
\mathbf{1 0 \mathbf { m M }}\end{array}$ & $\begin{array}{c}\text { Fludarabine } \\
\mathbf{2 5 m M}\end{array}$ \\
\hline$P>0.9999$ & $P=0.0837$ & $P=0.0309$ & $P=0.0320$ & $P=0.0053$ \\
\hline$P>0.9999$ & $P=0.0737$ & $P=0.0001$ & $P=0.0004$ & $P<0.0001$ \\
\hline$P>0.9999$ & $P=0.9982$ & $P=0.2043$ & $P=0.4756$ & $P=0.2742$ \\
\hline
\end{tabular}

D

$48 \mathrm{~h}$

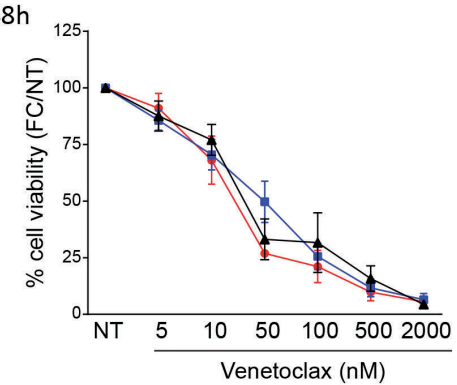

\begin{tabular}{|c|c|c|c|c|c|c|c|}
\cline { 2 - 8 } \multicolumn{1}{c|}{} & NT & $\begin{array}{c}\text { Venetoclax } \\
\mathbf{5 n M}\end{array}$ & $\begin{array}{c}\text { Venetoclax } \\
\mathbf{1 0 n M}\end{array}$ & $\begin{array}{c}\text { Venetoclax } \\
\mathbf{5 0 n M}\end{array}$ & $\begin{array}{c}\text { Venetoclax } \\
\mathbf{1 0 0 n M}\end{array}$ & $\begin{array}{c}\text { Venetoclax } \\
\mathbf{5 0 0 n M}\end{array}$ & $\begin{array}{c}\text { Venetoclax } \\
\mathbf{2 0 0 0 n M}\end{array}$ \\
\hline $\begin{array}{c}\text { TP53-M vs. } \\
\text { BIRC3-M }\end{array}$ & $P>0.9999$ & $P=0.9512$ & $P=0.5282$ & $P=0.9119$ & $P=0.4524$ & $P=0.7524$ & $P=0.7524$ \\
\hline $\begin{array}{c}\text { TP53-M vs. } \\
\text { WT }\end{array}$ & $P>0.9999$ & $P=0.9800$ & $P=0.6789$ & $P=0.3778$ & $P=0.7438$ & $P=0.8597$ & $P=0.8597$ \\
\hline $\begin{array}{c}\text { BIRC3-M } \\
\text { vs. WT }\end{array}$ & $P>0.9999$ & $P=0.8844$ & $P=0.9547$ & $P=0.2959$ & $P=0.8518$ & $P=0.9722$ & $P=0.9722$ \\
\hline
\end{tabular}

Figure 4. Responses of primary cells lines to fludarabine and venetoclax. (A-D) Viability of BIRC3-mutated ( $\mathrm{n}=6$ patients, red line), TP53-mutated $(\mathrm{n}=8$ patients, black line) and wildtype ( $n=7$ patients, blue line) primary CLL cells treated with different concentrations of fludarabine for $24 \mathrm{~h}(\mathrm{~A})$ and $48 \mathrm{~h}$ (B) or venetoclax for 24 $\mathrm{h}(\mathrm{C})$ and $48 \mathrm{~h}(\mathrm{D})$. The pairwise $P$ values are listed in the tables below the respective figures. M, mutated; WT, wildtype; NT, not treated. 
are addicted to the non-canonical NF- $\mathrm{BB}$ pathway, and that BIRC3-mutated CLL are resistant to fludarabine both in vitro and in patients. It still remains to be clarified whether NF-kB activation is the only molecular pathway that causes chemo-refractoriness in BIRC3-mutated CLL or whether other mechanisms are also involved. ${ }^{2429}$

Table 1. Clinical data of FCR-treated chronic lymphocytic leukemia patients according to BIRC3 mutational status.

\begin{tabular}{|c|c|c|c|c|}
\hline Characteristics & Total & Number of patients (\%) & BIRC3 mutated patients (\%) & BIRC3 wildtype patients (\%) \\
\hline $\begin{array}{l}\text { Male } \\
\text { Female }\end{array}$ & $\mathrm{N}=287$ & $\begin{array}{l}198(69.0 \%) \\
89(31.0 \%)\end{array}$ & $\begin{array}{c}5(55.6 \%) \\
4(44.4)\end{array}$ & $\begin{array}{l}193(69.4 \%) \\
85(30.6 \%)\end{array}$ \\
\hline $\begin{array}{l}\text { Binet A } \\
\text { Binet B-C }\end{array}$ & $\mathrm{N}=287$ & $\begin{array}{c}33(11.5 \%) \\
254(88.5 \%)\end{array}$ & $\begin{array}{l}1(11.1 \%) \\
8(88.9 \%)\end{array}$ & $\begin{array}{c}32(11.5 \%) \\
246(88.5 \%)\end{array}$ \\
\hline $\begin{array}{l}\text { IGHV mutated } \\
\text { IGHV unmutated }\end{array}$ & $\mathrm{N}=280$ & $\begin{array}{l}100(35.7 \%) \\
180(64.3 \%)\end{array}$ & $\begin{array}{c}0(0 \%) \\
9(100 \%)\end{array}$ & $\begin{array}{l}100(36.0 \%) \\
171(61.5 \%)\end{array}$ \\
\hline $\begin{array}{l}\text { 17p deletion } \\
\text { No 17p deletion }\end{array}$ & $\mathrm{N}=274$ & $\begin{array}{c}13(4.7 \%) \\
261(95.3 \%)\end{array}$ & $\begin{array}{c}0(0 \%) \\
9(100 \%)\end{array}$ & $\begin{array}{c}13(4.7 \%) \\
252(90.6 \%)\end{array}$ \\
\hline $\begin{array}{l}\text { 11q deletion } \\
\text { No 11q deletion }\end{array}$ & $\mathrm{N}=273$ & $\begin{array}{c}47(17.2 \%) \\
226(82.8 \%)\end{array}$ & $\begin{array}{c}5(55.6 \%) \\
4(44.4)\end{array}$ & $\begin{array}{c}42(15.1 \%) \\
222(79.9 \%)\end{array}$ \\
\hline $\begin{array}{l}13 q \text { deletion } \\
\text { No } 13 q \text { deletion }\end{array}$ & $\mathrm{N}=273$ & $\begin{array}{l}111(40.7 \%) \\
162(50.3 \%)\end{array}$ & $\begin{array}{l}3(33.3 \%) \\
6(66.6 \%)\end{array}$ & $\begin{array}{l}108(38.8 \%) \\
156(56.1 \%)\end{array}$ \\
\hline $\begin{array}{l}\text { Trisomy } 12 \\
\text { No trisomy } 12\end{array}$ & $\mathrm{~N}=272$ & $\begin{array}{c}50(18.4 \%) \\
222(81.6 \%)\end{array}$ & $\begin{array}{l}4(44.4 \%) \\
5(55.6 \%)\end{array}$ & $\begin{array}{c}46(16.5 \%) \\
217(78.1 \%)\end{array}$ \\
\hline
\end{tabular}

\begin{tabular}{lc}
\hline Median follow-up (years) & 6.8 \\
Median PFS (years) & 4.6 \\
\hline PFS \% (7 years) & $31.0 \%$ \\
Median OS (years) & 11.7 \\
\hline OS \% (7 years) & $75.5 \%$ \\
\hline
\end{tabular}

PFS: progression-free survival; OS: overall survival; IGHV: immunoglobulin heavy variable gene.

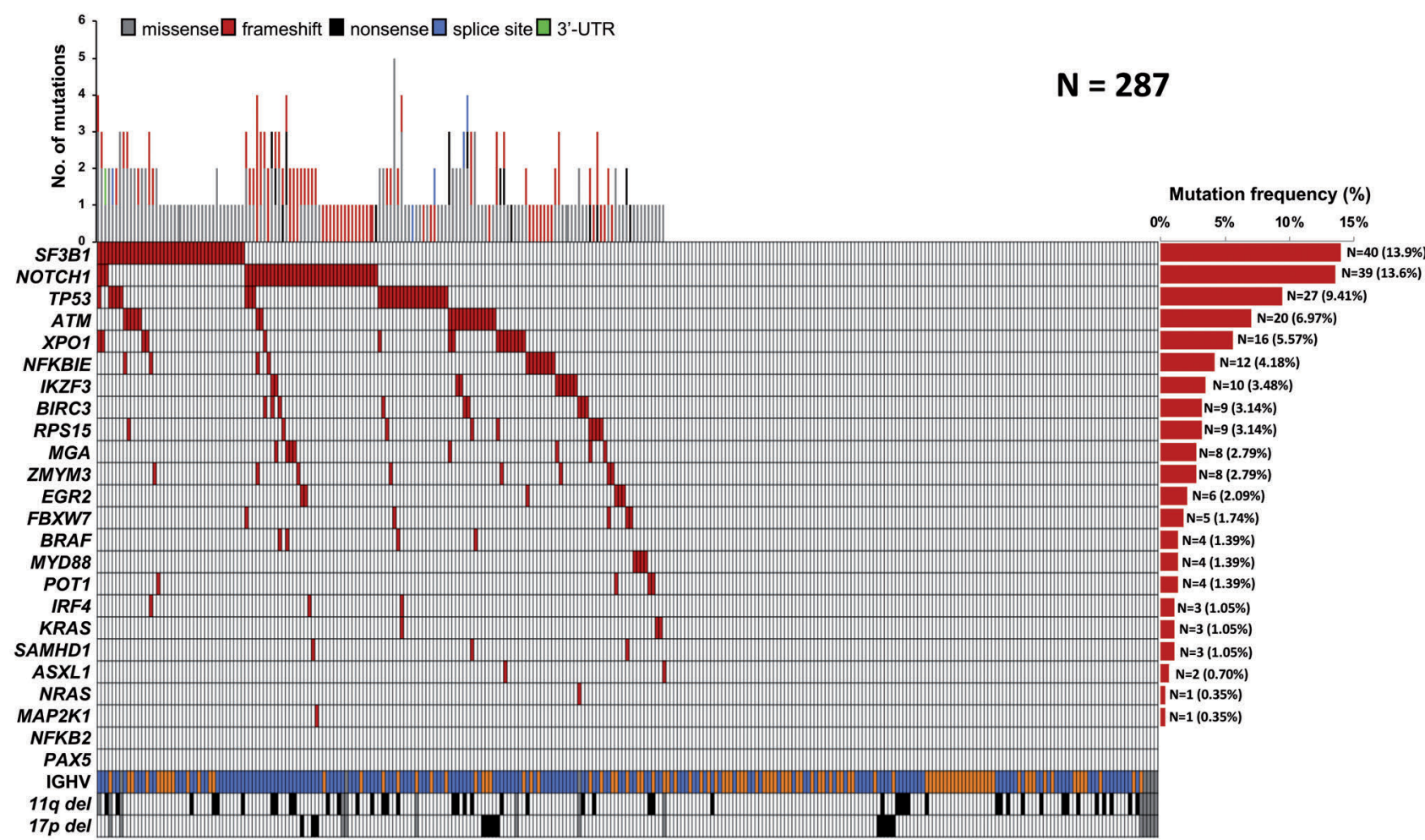

$\square$ Mutated IGHV $\quad \square$ Unmutated IGHV $\quad \square$ 17p/11q deleted $\square$ 17p/11q del and IGHV status not assessed

Figure 5. Mutational profile of the FCR-treated cohort. Case-level mutational profiles of 287 patients treated with FCR (fludarabine, cyclophosphamide, rituximab). Each column represents one tumor sample, each row represents one gene. The fraction of tumors with mutations in each gene is plotted on the right. The number and type of mutations in each patient are plotted above the heatmap. Mutations are highlighted in red. IGHV mutational status, 17p deletion and 11q deletion are plotted at the bottom of the heatmap. 
The introduction of FCR was a breakthrough in the management of young, fit CLL patients, leading to improvements in both PFS and overall survival compared to those achieved with previous treatment regimens. In both clinical trials and real-life cohorts, ${ }^{10-12}$ IGHV mutation status and TP53 disruption emerged as strong predictors of poor response to FCR. However, these molecular biomarkers do not fully capture all high-risk patients destined to relapse. We propose BIRC3 mutations as a new biomarker for the identification of patients at high risk of FCR failure, similarly to cases harboring TP53 disruption. If validated in independent series, BIRC 3 mutations may turn out as a new molecular predictor of FCR resistance that could be used to select patients to be treated with novel targeted agents.

Table 2. Univariate and multivariate analyses of progression-free survival.

\begin{tabular}{|c|c|c|c|c|c|c|c|c|c|c|c|c|c|}
\hline \multirow[b]{2}{*}{ Characteristics } & \multirow[b]{2}{*}{$\begin{array}{c}\text { 7-y PFS } \\
(\%)\end{array}$} & \multicolumn{4}{|c|}{ Univariate analysis } & \multicolumn{4}{|c|}{ Multivariate analysis } & \multicolumn{4}{|c|}{ Internal bootstrapping validation } \\
\hline & & $\begin{array}{l}\text { Median } \\
\text { PFS (y) }\end{array}$ & $95 \%$ Cl & $P$ & $\boldsymbol{P}^{*}$ & HR & LCI & UCI & $\mathbf{P}$ & HR & LCI & UCI & $\begin{array}{l}\text { Bootstrapping } \\
\text { selection (\%) }\end{array}$ \\
\hline Binet A & $40.3 \%$ & 4.5 & $2.4-6.6$ & 0.356 & - & - & - & - & - & - & - & - & - \\
\hline Binet B-C & $30.0 \%$ & 4.6 & $3.8-5.4$ & & & - & - & - & & - & - & - & \\
\hline IGHV mutated & $49.3 \%$ & 6.5 & $3.8-9.2$ & $<0.001$ & 0.003 & - & - & - & 0.001 & - & - & - & $98.8 \%$ \\
\hline IGHV unmutated & $23.0 \%$ & 3.9 & $3.5-4.4$ & & & 1.8 & 1.3 & 2.6 & & 1.9 & 1.3 & 2.7 & \\
\hline No 11q deletion & $33.4 \%$ & 5.0 & $4.2-5.9$ & 0.025 & 0.700 & - & - & - & - & - & - & - & - \\
\hline 11q deletion & $13.9 \%$ & 3.6 & $2.4-4.9$ & & & - & - & - & & - & - & - & \\
\hline No $17 p$ deletion & $33.0 \%$ & 4.8 & $4.1-5.6$ & $<0.0001$ & $<0.0001$ & - & - & - & $<0.0001$ & - & - & - & $99.5 \%$ \\
\hline 17p deletion & $\mathrm{nr}$ & 1.1 & $0-2.6$ & & & 4.0 & 2.2 & 7.5 & & 4.9 & 2.5 & 9.8 & \\
\hline TP53 wildtype & $33.8 \%$ & 5.4 & $4.3-5.8$ & $<0.0001$ & $<0.001$ & - & - & - & 0.030 & - & - & - & $73.3 \%$ \\
\hline TP53 mutated & $\mathrm{nr}$ & 2.8 & $2.0-3.5$ & & & 1.7 & 1.1 & 2.8 & & 1.8 & 1.1 & 3 & \\
\hline$B I R C 3$ wildtype & $32.2 \%$ & 4.8 & $4.1-5.6$ & $<0.001$ & 0.005 & - & - & - & 0.004 & - & - & - & $91.1 \%$ \\
\hline BIRC3 mutated & $\mathrm{nr}$ & 2.2 & $0.9-3.5$ & & & 2.8 & 1.4 & 5.6 & & 3.4 & 1.6 & 7.3 & \\
\hline EGR2 wildtype & $31.5 \%$ & 4.7 & $3.9-5.4$ & 0.015 & 0.420 & - & - & - & - & - & - & - & - \\
\hline$E G R 2$ mutated & $\mathrm{nr}$ & 1.5 & $0-3.8$ & & & - & - & - & & - & - & - & \\
\hline ATM wildtype & $32.5 \%$ & 4.8 & $4.1-5.6$ & 0.029 & 0.812 & - & - & - & - & - & - & - & - \\
\hline ATM mutated & $\mathrm{nr}$ & 3.2 & $2.4-4.1$ & & & - & - & - & & - & - & - & \\
\hline
\end{tabular}

y:year; P: P-value; $\mathrm{P}^{*}$ : Bonferroni correction; PFS: progression-free survival; CI: confidence interval; HR: hazard ratio; LCI: lower boundary of the confidence interval; UCI: upper boundary of the confidence interval; IGHV: immunoglobulin heavy variable gene; nr: not reached.

A

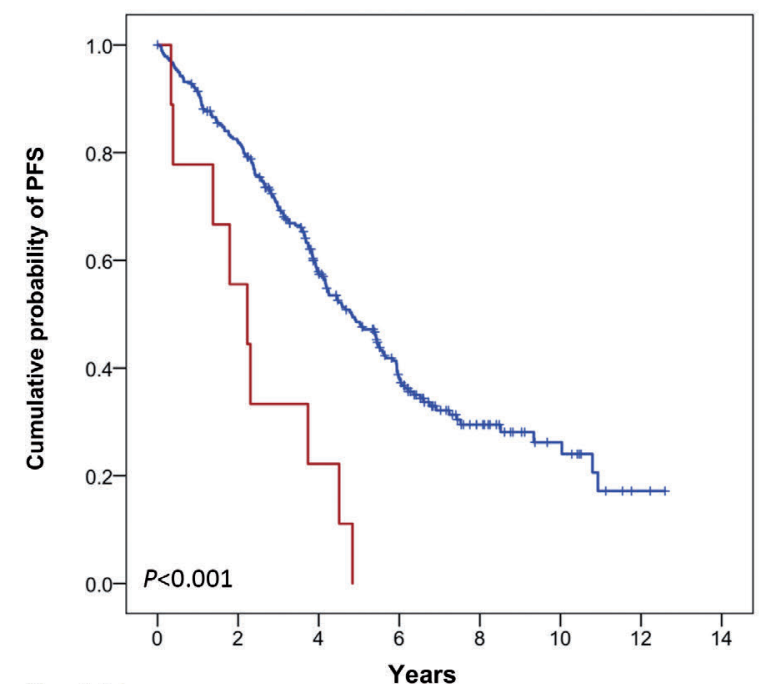

No. at risk

\begin{tabular}{|c|c|c|c|c|c|c|}
\hline BIRC3 wt $=278$ & 221 & 135 & 75 & 27 & 12 & 2 \\
\hline BIRC3 mut $=9$ & 5 & 2 & 0 & 0 & 0 & 0 \\
\hline
\end{tabular}

B

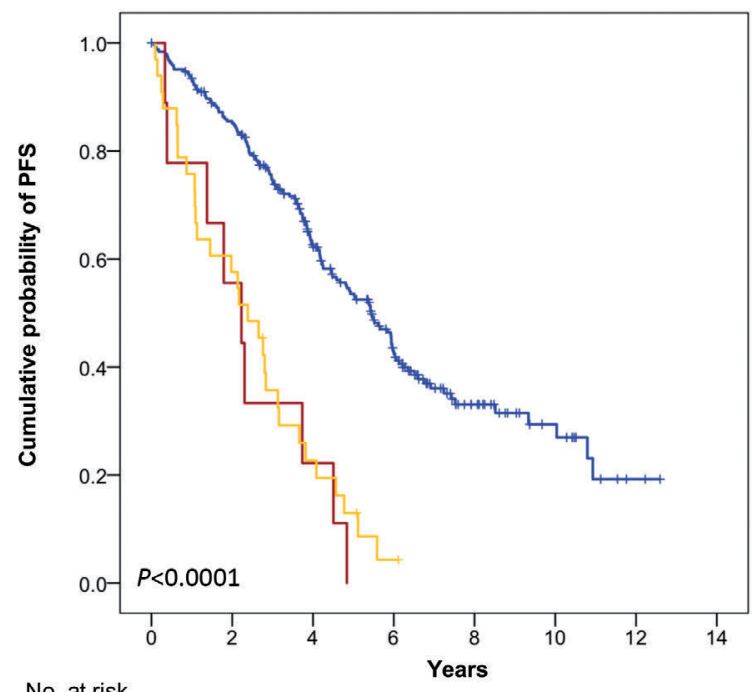

No. at risk

$\begin{array}{rcccccccc}\text { BIRC3 } / \text { TP53 wt } & =245 & 202 & 128 & 45 & 27 & 12 & 2 & 0 \\ \text { TP53 dis } & =33 & 19 & 7 & 1 & 0 & 0 & 0 & 0 \\ \text { BIRC3 mut }= & 9 & 5 & 2 & 0 & 0 & 0 & 0 & 0\end{array}$

Figure 6. Kaplan-Meier estimates of progression-free survival in BIRC3-mutated patients. (A) Cases harboring BIRC3 mutations are represented by the red line. BIRC3-wildtype cases are represented by the blue line. (B) Cases harboring BIRC3 mutations are represented by the red line. Cases harboring TP53 disruption (including TP53 mutation and/or 17p deletion) are represented by the yellow line. Patients devoid of BIRC3 mutation and TP53 disruption are represented by the blue line. 
Non-canonical NF- $\mathrm{BB}$ activation by BIRC3 mutations bypasses the block of BTK by ibrutinib. Consistently, NF$\kappa \mathrm{B}$ activation and cell survival are unaffected by ibrutinib in both CLL cells (our study) and mantle cell lymphoma cells. ${ }^{14}$ If this preclinical evidence is validated in ibrutinibtreated patients, BIRC3 mutations may also translate into a biomarker for informing selection of novel agents.

\section{Acknowledgments}

This work was supported by: Molecular bases of disease dissemination in lymphoid malignancies to optimize curative therapeutic strategies, (5 x 1000 n. 21198), Associazione Italiana per la Ricerca sul Cancro Foundation, Milan, Italy (grants to GG and RF) and Progetti di Rilevante Interesse Nazionale (PRIN),
(2015ZMRFEA), Rome, Italy; partially funded by the AGING Project - Department of Excellence - DIMET, Università del Piemonte Orientale, Novara, Italy; partially funded by Novara AIL ONLUS, Novara, Italy; Associazione Italiana per la Ricerca sul Cancro (AIRC IG-17314); Swiss Cancer League, ID HSR4660-11-2018, Bern, Switzerland; Research Advisory Board of the Ente Ospedaliero Cantonale, Bellinzona, Switzerland; European Research Council (ERC) Consolidator grant CLLCLONE ID: 772051; grant n. 320030_169670/1 Swiss National Science Foundation, Berne, Switzerland; Fondazione Fidinam, Lugano, Switzerland; Nelia \& Amadeo Barletta Foundation, Lausanne, Switzerland; Fond'Action, Lausanne, Switzerland; Translational Research Program, grant n. 6594-20, The Leukemia \& Lymphoma Society, New York, USA.

\section{References}

1. Mansouri L, Papakonstantinou N, Ntoufa S, Stamatopoulos K, Rosenquist R. NF- $\mathrm{kB}$ activation in chronic lymphocytic leukemia: a point of convergence of external triggers and intrinsic lesions. Semin Cancer Biol. 2016; 39:40-48

2. Bonizzi G, Karin M. The two NF-kappaB activation pathways and their role in innate and adaptive immunity. Trends Immunol. 2004:25(6):280-288

3. Oeckinghaus A, Hayden MS, Ghosh S. Crosstalk in NF-KB signaling pathways. Nat Immunol. 2011:12(8):695-708.

4. Sun SC. The noncanonical NF-kB pathway. Immunol Rev. 2012;246(1):125-140

5. Asslaber D, Wacht N, Leisch M, Oi Y, et al. BIRC3 expression predicts CLL progression and defines treatment sensitivity via enhanced NF- $\mathrm{kB}$ nuclear translocation. Clin Cancer Res. 2019;25(6):1901-1912.

6. Puente XS, Beà S, Valdés-Mas R, et al. Noncoding recurrent mutations in chronic lymphocytic leukaemia. Nature. 2015;526 (7574):519-524.

7. Landau DA, Tausch E, Taylor-Weiner AN, et al. Mutations driving CLL and their evolution in progression and relapse. Nature. 2015:526(7574):525-530

8. Hallek M, Cheson BD, Catovsky D, et al. Guidelines for diagnosis, indications for treatment, response assessment and supportive management of chronic lymphocytic leukemia. Blood. 2018;131(25):2745-2760.

9. Gaidano G, Rossi D. The mutational landscape of chronic lymphocytic leukemia and its impact on prognosis and treatment. Hematology Am Soc Hematol Educ Program. 2017;2017(1):329-337.

10. Rossi D, Terzi-di-Bergamo L, De Paoli L, et al. Molecular prediction of durable remission after first-line fludarabine-cyclophosphamide-rituximab in chronic lymphocytic leukemia. Blood. 2015;126(16):1921-1924.
11. Thompson PA, Tam CS, O'Brien SM, et al Fludarabine, cyclophosphamide, and rituximab treatment achieves long-term diseasefree survival in IGHV-mutated chronic lymphocytic leukemia. Blood. 2016;127(3):303309.

12. Fischer K, Bahlo J, Fink AM, et al. Long-term remissions after FCR chemoimmunotherapy in previously untreated patients with CLL: updated results of the CLL8 trial. Blood. 2016;127(2):208-215

13. Rossi D, Fangazio M, Rasi S, et al. Disruption of BIRC3 associates with fludarabine chemorefractoriness in TP53 wild-type chronic lymphocytic leukemia. Blood. 2012;119(12):2854-2862.

14. Rahal $R$, Frick $M$, Romero $R$, et al. Pharmacological and genomic profiling identifies NF-kB-targeted treatment strategies for mantle cell lymphoma. Nature Medicine. 2014.20(1):87-92

15. Hallek M, Fischer K, Fingerle-Rowson G, et al. Addition of rituximab to fludarabine and cyclophosphamide in patients with chronic lymphocytic leukaemia: a randomised, open-label, phase 3 trial. Lancet. 2010:376(9747):1164-1174.

16. Stilgenbauer S, Schnaiter A, Paschka P, et al. Gene mutations and treatment outcome in chronic lymphocytic leukemia: results from the CLL8 trial. Blood. 2014;123(21):32473254 .

17. Grossmann V, Kohlmann A, Schnittger A, et al. Recurrent ATM and BIRC3 mutations in patients with chronic lymphocytic leukemia (CLL) and deletion 11q22-q23. Blood. 2012;120(21):1771

18. Rose-Zerilli MJ, Forster J, Parker $\mathrm{H}$, et al. ATM mutation rather than BIRC3 deletion and/or mutation predicts reduced survival in 11q-deleted chronic lymphocytic leukemia: data from the UK LRF CLL4 trial. Haematologica. 2014;99(4):736-742.

19. Baliakas P, Hadzidimitriou A, Sutton LA, et al. Recurrent mutations refine prognosis in chronic lymphocytic leukemia. Leukemia. 2015;29(2):329-336

20. Nadeu F, Delgado J, Royo C, et al. Clinical impact of clonal and subclonal TP53, SF3B1, BIRC3, NOTCH1, and ATM mutations in chronic lymphocytic leukemia. Blood. 2016;127(17):2122-2130.

21. Vince JE, Wong WW, Khan N, et al. IAP antagonists target cIAP1 to induce TNFalpha-dependent apoptosis. Cell. 2007;131(4):682-693.

22. Jost PJ, Ruland J. Aberrant NF-kappaB signaling in lymphoma: mechanisms, consequences, and therapeutic implications. Blood. 2007;109(7):2700-2707.

23. Raponi S, Del Giudice I, Ilari C, et al. Biallelic BIRC3 inactivation in chronic lymphocytic leukaemia patients with 11q deletion identifies a subgroup with very aggressive disease. Br J Haematol. 2019;185(1):156-159.

24. Hewamana S, Lin TT, Jenkins $C$, et al, The novel nuclear factor-kappaB inhibitor LC-1 is equipotent in poor prognostic subsets of chronic lymphocytic leukemia and shows strong synergy with fludarabine. Clin Cancer Res. 2008;14(24):8102-8111.

25. Hewamana S, Alghazal S, Lin TT, et al. The NF-kB subunit Rel A is associated with in vitro survival and clinical disease progression in chronic lymphocytic leukemia and represents a promising therapeutic target. Blood. 2008;111:4681-4689.

26. Beg AA, Baltimore D. An essential role for NF- $\mathrm{KB}$ in preventing TNF- $\alpha$-induced cell death. Science. 1996;274:782-784.

27. Wang CY, Mayo MW, Baldwin AS, Jr. TNFand cancer therapy-induced apoptosis: potentiation by inhibition of NF-KB. Science. 1996;274:784-787.

28. Webster GA, Perkins ND. Transcriptional cross talk between NF- $\mathrm{kB}$ and $\mathrm{p53}$. Mol Cell Biol. 1999:19:3485-3495.

29. Nakanishi C, Toi M. Nuclear factor-кB inhibitors as sensitizers to anticancer drugs. Nat Rev. 2005;5:297-309. 